"New Strategies for Designing Inexpensive but Selective Bioadsorbants for Environmental Pollutants: Selection of Specific Ligands and their Cell Surface Expression."

\title{
Brent Iverson
}

Period covering 9/15/96 - 9/14/97

Department of Chemistry and Biochemistry

The University of Texas at Austin

Austin, TX 78712

Grant DE-FG07-96ER62322, And MOO1

\section{Technical Progress Report}

Progress for the last twelve months has revolved around setting up our antibody engineering and surface display system for use with a metal-complex binding antibody.

1) We have isolated genes for the $V$ regions of the heavy and light chains for the Ru(bpy)3 specific monoclonal AC1106 (Shreder, K S., Hariman, A., and Iverson, B.L., J. Am. Chem. Soc. 1996, 118, 3192-3201). This antibody binds Ru(bpy), derivatives with better than nanomolar affinity, and will serve as our generic metalcomplex binding pocket. Cloning antibody genes from hybridomas is complicated by the fact that primers must be found that amplify the particular heavy and light chain genes in the hybridoma of interest. Antibody gene amplification primers are generally designed to amplify antibody repertoires from lymphocyte mRNA isolated from animals. While cloning repertoires from animals is routinely successful due to the diverse population of target $\mathrm{m}$ RNA, hybridomas have a single target sequence. Therefore, multiple primers and conditions must be tried before the correct primer combination is identified. 
2) A -((gly),ser),- linker was added between the heavy and light chain using SOE-PCR.

3) The entire scFv construct has been ligated into pGemT using a T-vector cloning strategy.

4) More recently. the scFv has been cloned into a pET25b(+) vector for soluble expression.

5) We are in the middle of the synthesis of various metal complexes to be used for a thorough binding analysis of the soluble scFv (mentioned in 6) below).

6) We are preparing milligram quantities of anti-Ru(bpy $)_{3} \mathrm{scFv}$ in preparation for the detailed characterization in solution using the metal complexes mentioned in 5) above.

7) Preliminary ELISA data has now indicated that the AC1106 scFv is active! ! ! !

8) We have begun cloning the AC1106 $\mathrm{scFv}$ into our bacterial surface expression system in preparation for antibody engineering efforts. By using iterative rounds of randomization and selection using via fluorescence activated cell sorting (FACS) the affinities for different metal ions will be evolved.

9) In advances related to the proposed work, we have been able to optimize further the efficiency of our bacterial surface expression/FACS selection system for isolating interesting new antibodies. Using the optimized protocols on an $\mathrm{scFv}$ that binds the heart glycoside digoxin, we were able to produce an improved $\mathrm{scFv}$ with subnanomolar affinity after a single round of selection! These powerful new procedures will be employed in the upcoming metal-complex binding antibody evolution studies. 


\section{Budget Information}

Contract and budget period 9/14/96 to $9 / 14 / 99$.

$\$ 749,392 \quad$ Money received on $9 / 25 / 96$

- 161.447 In direct costs

$\$ 587,945$

- 111,558 Iverson/Georgiou money spent as of 9/30/97 - YEAR ONE

- 73,871 Subcontract (Univ. of Pitts.) money spent - YEAR ONE 402,516 Balance remaining as of 9/30/97

$-215,176$ Subcontract monev remaining of Univ. of Pittshurgh

$\$ 187,340 \quad$ Remaining money at UT for Iverson/Georgiou

YEAR TWO projected spending: Georgiou summer salary $\$ 7350$

Iverson summer salary $\$ 5670$

Post Doc 1

Post Doc $2 \quad \$ 26,200$

Fringes $\$ 17,663$

Supplies $\$ 15,000$

Travel $\$ \$ 2500$

\begin{tabular}{lll}
$-1 \mathrm{PQ} .583 \quad$ TOTAL & $\frac{\$ 2.583}{100.583}$ \\
\hline
\end{tabular}

YEAR THREE projected spending: Georgiou summer salary \$7718

Tverson summer salary \$5954

Post Doc 1

Post Doc $2 \quad \$ 26,270$

Fringes $\$ 17,877$

Supplies $\$ \$ .15 .000$

Travel $\$ \$, 500$

\begin{tabular}{lll}
-101.589 & TOTAL & $\frac{\$, 500}{101.589}$ \\
\hline
\end{tabular}

- 14,833 Balance remaining after 9/14/99 end of contract period. 